\title{
New Technological Settings of Communication to Improve the Traditional Techniques in Clinical Psychology and Psychotherapy
}

\author{
Gianluca Castelnuovo*
}

Istituto Auxologico Italiano IRCCS, Psychology Research Laboratory, San Giuseppe Hospital, Verbania, Italy and Department of Psychology, Catholic University of Milan, Italy

\begin{abstract}
Internet and new communication technologies have achieved a rapid development in the last years producing a significative impact on clinical psychology and psychotherapy. Psychotherapists seem to rely with more and more interest on the new technological tools such as telemedicine, audio and video chats, e-mail, SMS, MMS and the Instant Messaging Tools (IMs). All these technologies outline a stimulating as well as complex scenario: in order to effectively exploit their potential, it is important to study which is the possible role played by the Internet-based tools inside a psychotherapeutic or psychological treatment. The main goal of this article is to provide a framework for the integration of new technological tools and settings in mental health care. Different theoretical positions about the possible role played by Internet based therapy (e-therapy) are reported showing the possible changes that psychotherapy will necessarily cope with.
\end{abstract}

\section{INTRODUCTION}

In the last years the widespread presence of user friendly computers allows more and more people and professionals (clinical psychologists, psychotherapists, etc.) to use Internet and other technological tools in the field of mental health treatment. The rapid development of Internet and new communication technologies is having a growing impact on clinical settings, overall on clinical psychology and psychotherapy.

In spite of this present scenario, a survey on a sample of 1000 psychologists selected from APA (American Psychological Association) has shown that only $2 \%$ of respondents (596 practitioners with the median age of 50, ranging from 33 to 72 ) had used Internet-based technologies in the delivery of health care (VandenBos and Williams, 2000). Even if nowadays the integration between "techno" and "psycho" is still far, more and more psychologists are trying to use these technologies into their practice and research (Bang, Timpka, Eriksson, Holm, \& Nordin, 2007; Bara-Carril et al. 2004; Boydell, Volpe, Kertes, \& Greenberg, 2007; Castelnuovo et al. 2004; Castelnuovo, Gaggioli, Mantovani, \& Riva, 2003; Frueh, Monnier, Grubaugh et al. 2007; Frueh, Monnier, Yim et al. 2007; Heinicke, Paxton, McLean, \& Wertheim, 2007; Lester, 2006; Ljotsson et al. 2007; Looi \& Raphael, 2007; Mohr, Hart, \& Vella, 2007; Molinari \& Riva, 2004; Myers, Swan-Kremeier, Wonderlich, Lancaster, \& Mitchell, 2004; Naylor, Keefe, Brigidi, Naud, \& Helzer, 2008; Norcross, Hedges, \& Prochaska, 2002; O'Reilly et al. 2007; Oakes, Battersby, Pols, \& Cromarty, 2008; Park, 2006; Postel, de Jong, \& de Haan, 2005; Rawabdeh, 2007; P. Recupero \& Rainey, 2005; P. R. Recupero \& Rainey, 2006; Riva, 2005; Riva, Preziosa, Grassi, \& Villani, 2006; Robinson \& Serfaty,

Address correspondence to this author at the Istituto Auxologico Italiano IRCCS, Psychology Research Laboratory, San Giuseppe Hospital, Verbania, Italy and Department of Psychology, Catholic University of Milan, Italy; Tel/Fax: +39-0323514338;

E-mails: gianluca.castelnuovo@auxologico.it;

gianluca.castelnuovo@unicatt.it
2003, 2008; Shepherd et al. 2006; Sherwood et al. 2006; Silk \& Yager, 2003; Sulzbacher, Vallin, \& Waetzig, 2006; Todder \& Kaplan, 2007; Ugarriza \& Schmidt, 2006; Urness, Wass, Gordon, Tian, \& Bulger, 2006; Wangberg, Gammon, \& Spitznogle, 2007; Wiederhold \& Wiederhold, 2003; Williams, 2003; Yager, 2003; Yellowlees, Hilty, Marks, Neufeld, \& Bourgeois, 2008; Zabinsky, Celio, Joy Jacobs, Manwaring, \& Wilfley, 2003).

Stamm (1998) observes that "psychologists do not have to become technology specialists to be competent providers of telehealth services... However, to best know when and how to use technology to support healing... psychologists will need more technology proficiency, particularly with computers, than has been the norm. This is particularly true for those who will be establishing their practices in the coming decades." (pp. 536-537).

To help psychologists and other professionals to learn new competencies for expanding their practice in telehealth field, self assessment questionnaires, guidelines and training programs are nowadays available (Barak, 1999; Glueckauf, Pickett, Ketterson, Loomis, \& Rozensky, 2003).

Psychotherapists seem to rely not only on the traditional telephone (Reese, Conoley, \& Brossart, 2002), but also on the new technological tools such as videophone, audio and video chat, e-mail (Norcross et al. 2002), SMS (Bauer, Percevic, Okon, Meermann, \& Kordy, 2003) and the new Instant Messaging Tools (IMs)(Castelnuovo, Gaggioli, \& Riva, 2001). In fact in 2001 the demand for health information online has been carried on by 100 millions of individuals in the United States (Taylor \& Leitman, 2001).

In these changing clinical settings new terms have been coined to identify this fruitful integration between "technology" and "psychology-medicine": telehealth is the use of telecommunications and information technologies to provide access to health information, assessment, diagnosis, intervention, consultation, supervision, education and follow-up programs across geographical distance (Glueckauf et al. 
2003; Glueckauf, Whitton, \& Nickelson, 2002; Liss, Glueckauf, \& Ecklund-Johnson, 2002; Nickelson, 1998).

To stress the real added value and advantage of using Internet-based tools in clinical psychology, according to Glueckauf et al. (2003), we report here four main reasons.

The possibility to provide health information and services across geographical distance for underserved population.

Internet can be the only possibility to allow the provision of appropriate health assistance in remote areas where specialized staff and facilities are not widespread: the AKAMAI Telemedicine Program, in the case of Hawai, and Alaska Telemedicine Program, in the case of Alaska, are two examples (Stamm, 1998). Glueckauf et al. (2003) notes that "a wide array of telehealth services is currently offered across diverse settings, including hospitals, community mental health centers, long-term care facilities, schools, prisons, and rural health centers" (p. 160).

The possibility to increase the quality of health information and services in particular areas or for specific populations.

Ensuring health-services "on-line" could also reduce gaps of quality in treatments between different demographic groups (Shortliffe, 2000), traditionally without the same possibility to reach Health-care organizations: there are considerable differences in the access to psychological services in the world. But opponents of e-therapy, as noted by ManhalBaugus (2001) state that "it is limited to clients who are reasonably educated writers and readers" and "this leads to the issue of elitism. The use of literally techniques to conduct therapy may be relegated to a select group of educated, middle to upper class individuals"; however "real-life psychotherapy is usually based on average intellectual abilities and skills and more costly than online therapies" (p. 559).

The possibility to ensure a continuous medical and psychological service overall for chronic disabilities reducing the cost of an extended traditional assistance.

The real cost effectiveness of telehealth programs has only little evidences but it is very likely for extended treatments where an Internet-based interaction between a therapist and a patient could avoid a physical contact saving economical, social and individual costs. Some examples could be followed up therapeutic sessions or brief consultations about the state of progress of psychotherapies in advanced steps.

The growing trend of patients' preference towards accessing therapy via a home-based computer system.

The status of "being on-line" is coming "normal" and more and more individuals are carrying on daily life activities in the Web including the search of on-line psychological treatments.

Another key advantage of using Internet-based tools in mental health field is related to the improvement and facility of the communication between different practitioners who take charge of the same patient. Until now the telephone has played a central role in the process of negotiating how activities involved in the treatment of patients can be scheduled, but new Internet-based tools are more and more used in the collaboration between different members of the clinical staff. These tools could support communication, problem solving and clinical decision-making process providing therapists with the possibility to share different records, information and clinical materials.

So new Internet-based tools could improve the relationship between patients and therapists and between practitioners who are taking charge of the same patient. About the first issue, "extensions of teletherapy to people in restricted conditions such as prisons, hospices, nursing homes need to be studied" (Day and Schneider, 2002).

\section{TECHNOLOGY INTO PSYCHOTHERAPY: IS IT POSSIBLE AN INTEGRATION?}

Inside this new technological scenario, in order to effectively exploit its potential, it is important to study which is the possible role played by the Internet-based tools inside a psychotherapeutic treatment. Could the technology substitute the health care practitioners or are the tools only a resource in addition to the traditional ones in the therapist's hand?

According to Jerome and Zailor (2000), something in the clinical field will be altered in the presence of emerging technologies, so "it is thus important to study the impact of these changes as they occur, and it is imperative that new technological competencies be developed as clinicians integrate these technologies into their research and practice" (p. 478).

So the coming of $e$-therapy, defined by Grohol (1999) as "a new modality of helping people resolve life and relationship issues", opens a lot of questions about the status of "technology in psychotherapy". First of all, could psychotherapy be carried on using only technological tools without the traditional face-to-face interactions?

About this issue there are different theoretical positions that it is possible to summarize in five main ones:

Psychotherapy could be made on-line and e-therapy differs from traditional treatments simply for the use of a technological medium. According to this point of view, the methods, techniques and procedures related to a particular theory, model or approach used in psychotherapy are preserved and the only relevant difference is the change of setting from a f-2-f setting to an Internet-mediated one. Day and Shneider in a recent report examined the level of working alliance in three conditions (face-to-face, audio and video individual therapy) and noted that "the similarities among the three treatment groups - face-to-face, video teleconference and audio conference - came through more strongly than any differences" (p. 501)(Day and Schneider, 2002).

Psychotherapy could be made on-line but e-therapy differs from the traditional treatments because the communication suffers from critical changes. According to this position, communication by e-mail appears to completely lack the nonverbal cues and channel many of practitioners consider as an integral part of traditional approaches (Alleman, 2002). So some therapeutic scenarios, such as highly experiential setting and approaches that give great importance to the front-end diagnosis step, are strictly related to face-to-face situations and need to be deeply redesigned in case of using 
an Internet-based medium (Alleman, 2002). For some theorists the communication over the Internet would be an inadequate replacement for face-to-face intimacy (Robson and Robson, 1998).

Psychotherapy could be made on-line and e-therapy could be preferentially used with some patients. The absence of the nonverbal channel in cybertherapy has been traditionally perceived as a limit by practitioners but the difference between a f-2-f communication and an Internet-based one could be favorable for the technological scenario. Posture, tone, inflection, eye contact and other nonverbal cues can give the therapist a lot of precious information but can also overload, distract, confuse or intimidate the patient. E-mail contacts seem to be the way clients prefer to seek in spite of therapists' disapproval. Due to its "disinhibition effect" in comparison with face-to-face (Suler, 2001), cybertherapy could be an important choice for the treatment of anxiety disorders, at least in a first part of the therapy, during the creation of a functional working alliance. According to Day and Schneider (Day and Schneider, 2002), patients in etherapy make "more of an effort to communicate, taking more responsibility for the interaction than they did in faceto-face traditional therapy" (p. 502) or they could feel safer opening and telling more in a cyber situation in comparison with a traditional face-to-face setting. We have also to take into account that several psychological disorders result in extreme discomfort with human contact. "Agoraphobics, socially anxious and avoidant personalities often find close proximity with unfamiliar others intolerable. One possible tactic involves using telephone, video, and face-to-face communication with the psychotherapist as such a client gains trust and comfort" (p.502)(Day and Schneider, 2002). Also Alleman noted that "although more research is needed, it seems plausible that people could communicate effectively online even when the content of their communication is fraught with affective intensity" (p.503)(Alleman, 2002). According to this approach, Cohen and Kerr (Cohen and Kerr, 1998) found no significant differences in the effectiveness of the treatment between computer-mediated and faceto-face conditions with a sample of patients suffering from excessive anxiety(Cohen and Kerr, 1998).

Psychotherapy is something different from e-therapy and web counseling. Many authors differentiate the structured traditional clinical iter from a cyber therapeutic relationship (Grohol, 1999, Maheu and Gordon, 2000, Metanoia, 2001a). Online it is possible to provide assessment, counseling and advices to choose and start a treatment but the Internet-based communication does not allow a traditional individual, group or family psychotherapy. Grohol discusses that "it would be inappropriate to compare it (e-therapy) to traditional face-toface psychotherapy, assessment or traditional services" (Grohol, 1999, p. 1).

E-therapy could enhance traditional psychotherapy in some steps of it. For this point of view, cybertherapy is only a preliminary (assessment) of final (follow-up) step of the therapeutic iter, but the most important methods, techniques and procedures related to a particular theory, model or approach could be carried on only in a traditional face-to-face setting. So e-therapy could be only a popular and widespread medium to lead people to traditional and functional psychotherapy.

In my view, an emerging scenario could characterize the future clinical setting: old (and functional) practices could be integrated and enhanced through new (and promising) media. This framework aiming at matching "techno" and "psycho" for clinical purposes will be explained in the next section.

\section{A FRAMEWORK PROPOSAL TO INTEGRATE NEW TECHNOLOGICAL SETTINGS AND TOOLS WITH TRADITIONAL METHODS AND TECHNIQUES IN CLINICAL PSYCHOLOGY}

The most critical issue in this converging space between technology and psychology is to which extent the innovative hypermedia tools could influence, block, exceed or support the old and functional techniques and protocols in psychotherapy. Is an integration possible?

In our view the basic principles, methods, techniques and procedures in the clinical fields have not to be changed: $e$ therapy should not modify theories, techniques and methods typical of each approach (psychoanalytic, systemic, cognitive, behavioral, interpersonal, strategic, etc.) but could affect the level of communication and so the possible relationship and alliance between the therapist and the patient (see the Table 1).

The traditional setting could move into a cyber (or virtual, synthetic, etc.) world, without affecting the basic principles and methods of the therapeutic iter.

It is important to shed light on an emerging kind of Internet-based tools: the instant messaging (IM) tools. For the traditional tools (e-mail, chat, videoconference, etc.) please go to (Castelnuovo et al. 2001).

These tools can be described as "on-line setting where networks of multimedia nodes connected by links are used to present information and manage retrieval" (Federico, 1999, p. 662).

While a hypertext consists primarily of textual information, hypermedia includes multiple information formats, such as visual, musical and animation elements. When hypermedia are used as communication tools, they are defined instant messaging (IM) tools (Riva, 2001).

The real added value of IMs, in comparison with traditional Internet-based tools such as chat, e-mail, videoconference, etc., is that this medium further enhances the user's experience by the integration of different forms of computermediated-communication (e-mail, IRC etc.) into one fully integrated interface. IMs could integrate the communication potential offered by the Internet with the richness of different multimedia contents.

About the practical possibilities provided to patients and therapists, IMs could go further than the traditional Internetbased tools because IMs could create a new setting (a psychotherapist's virtual office) where the whiteboard could allow to communicate (using verbal and not-verbal languages), to exchange and share information (video files, document, etc.) with an open channel between clients and care givers. 
Table. 1. The Possible Role and Influence of Internet-based Tools on the Levels of Interaction Between Therapists and Patients

\section{LEVELS OF INTERACTION BETWEEN THERAPIST AND PA-} TIENT

1) Methods, techniques and procedures related to a particular theory, model or approach used in psychotherapy

EXAMPLE: In a cognitive approach some techniques are for example alternative interpretation, label shifting, cognitive reframing, etc.

DEGREE OF INFLUENCE OF INTERNET-BASED TOOLS ON THIS LEVEL: low

CONSIDERATIONS: The methods/techniques are dependent on theory/approach and not on the medium used. So a particular technique could be used in a face to face interaction as effectively as using a simple Internet-based tool.

GENERAL INDICATIONS FOR A FRUITFUL USE OF INTERNETBASED TOOLS IN PSYCHOTHERAPY: At this level the main suggestion is to use Internet-based tools that at least could ensure the basic rules of communication in order to allow patients to understand the indications provided by therapists.

\section{2) Communication}

EXAMPLE: The communication could be direct, indirect, evocative, hypnotic, more verbal or not-verbal oriented, etc...

DEGREE OF INFLUENCE OF INTERNET-BASED TOOLS ON THIS LEVEL: high

CONSIDERATIONS: The communication could be limited or enhanced according to the medium used. If an Internet-based tool, such as written chat, does not allow the use of not verbal language, the communication could be deeply compromised.

GENERAL INDICATIONS FOR A FRUITFUL USE OF INTERNETBASED TOOLS IN PSYCHOTHERAPY: It is necessary to allow a complete communication (verbal and not verbal), as much as possible, between therapist and patient. New Instant Messaging Tools are advised because they could ensure a wide communication due to their integration of different media and languages.

\section{3) Relationship}

EXAMPLE: Between a therapist and a patient there are different kinds of relationship according to the step of the therapy, the therapist's ability, the patient's resistance, etc.: so the possibilities among therapists and patients range between a lack of trust to an extreme confidence ("rapport") with a high level of therapeutic alliance.

DEGREE OF INFLUENCE OF INTERNET-BASED TOOLS ON THIS LEVEL: high

CONSIDERATIONS: The communication is one of the key factors in the creation of a good relationship and alliance between a therapist and a patient. So a limited communication could drive to a dysfunctional relationship.

GENERAL INDICATIONS FOR A FRUITFUL USE OF INTERNETBASED TOOLS IN PSYCHOTHERAPY: Internet-based tools could be introduced only after the creation of a "good-enough" relationship between therapist and patient that is typical of the first step of the treatment in a traditional setting. Moreover, during the others steps of the therapeutic iter, a verbal and not-verbal communication has to be ensured in order not to loose the previously built relationship. So new Instant Messaging Tools are advised.

As indicated in Figure 1, in a hypothetical continuum between complete absence and presence of communication in the interaction between a therapist and a patient, IMs could more and more move closer to "face to face" situations that still remain the ideal situation for psychotherapy. E-mail, chat, VTC seem to affect the communication between two users but cannot recreate the clinical setting across distance.

Different users, who are simultaneously browsing the same website, can communicate with each other sharing different kinds of files. Usually, an IM allows the user to conduct group and private chats, to exchange information and files, and browse the same web-pages. On any website, IM users can see a list of other users and talk with them on group and private levels. Furthermore, each user can obtain a constantly updated list of all the other online users who are visiting the same website (Riva, 2001).

Taken together, the results of this review suggest that instant messaging are well-suited for a telemedicine application in psychotherapy (Riva, 2000). However, the real effectiveness of such tools depends largely on the ability of therapists to coherently integrate the features of IMs in the clinical procedure.

For this reason, it would be useful to provide the reader with some insights regarding potential ways of using IMs in psychotherapy.

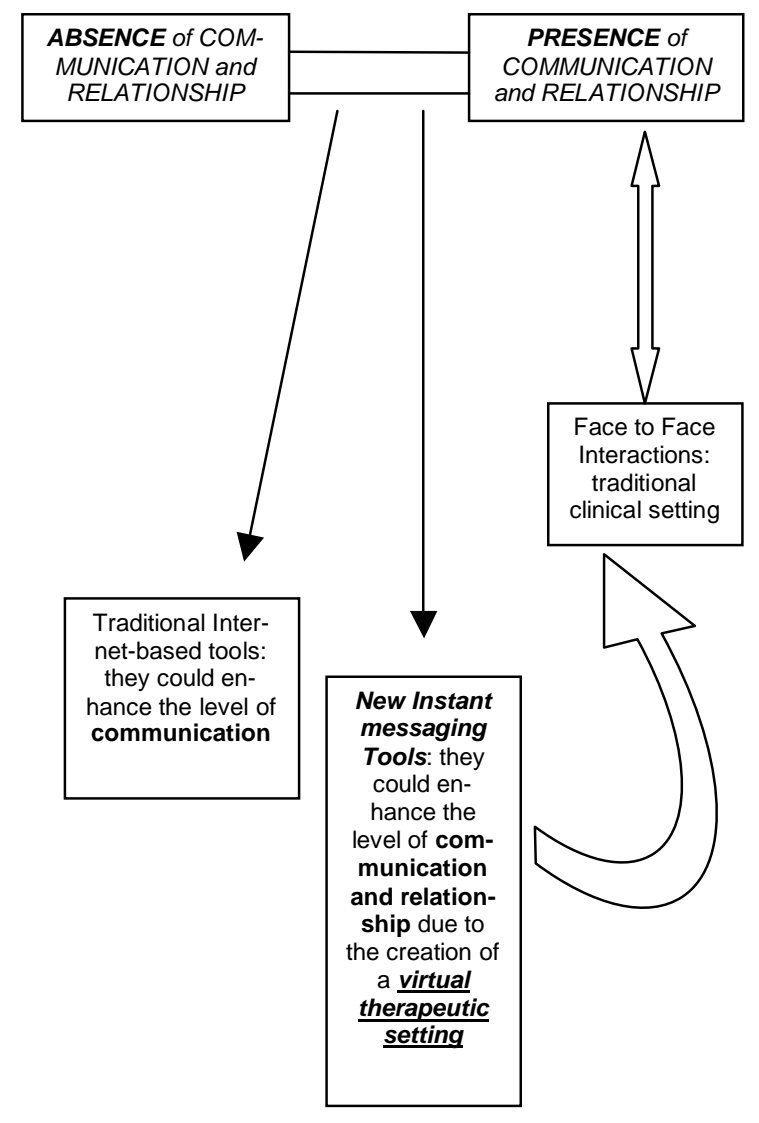

Fig. (1). The continuum between absence and presence of communication and relationship in a clinical setting and the possible position of IMs in comparison with traditional Internet-based tools.

The following considerations are not meant to be exhaustive, rather to stimulate the creativity and the curiosity of mental health professionals who are particularly interested in promoting new solutions for using Internet related technologies in the clinical practice.

By using IMs, the therapist and the patient are able to share pictures, screenshots and videos captured by a video camera installed on their workstations and to communicate their ideas and impressions using both the text chat and voice chat features. The "application sharing" feature enables the therapist to send to the patient a psychometric questionnaire for assessment purposes and receive the result in real time. 
Consequently, the therapist is able to provide the patient with a feedback in a fraction of the time that this process normally requires. So therapists can carry on parts of the clinical process, e.g., diagnosis, therapeutic sessions, follow up, etc, using these new tools with patients.

The "virtual whiteboard" is another interesting feature that has promising applications. For example, the therapist can use the whiteboard to enrich his/her explanations with diagrams and drafts, which can be exported to other applications.

Finally, the text-chat feature allows the therapist to save the content of the dialogue as text file, making it available for future reference.

Less complex IMs represent an acceptable compromise between simplicity of use and richness of communication features. Although few exceptions, IMs have a very intuitive and easy-to-learn interface. This last feature is very important, because it makes IMs well suited also for novice Internet users. Another advantage of most IMs is that therapist can send the patient a request for a communication session using either the IM feature, if the patient is logged in, or email. Once the session is open, the therapist can chat with the patient privately or in group, by sending a request to the other patients that are online at that moment. In some IMs, the users can rapidly move from the text chat to the voice chat, which has often a valuable quality. Furthermore, even using simple IMs applications the therapist is able to send the patients files (i.e. assessment questionnaires) and to receive the results even within the same session.

\section{CONCLUSION}

E-therapy could represent an useful integration between technological tools and traditional clinical techniques and protocols in order to improve the effectiveness and efficiency of psychotherapy. In the future psychotherapists and psychologists will probably use not only telephone and email (currently the most widespread telehealth instruments), but also tools (such as instant messaging tools) that support advanced communicational features such as real time video and audio connections, exchange of text and video messages etc. (Castelnuovo et al. 2001; Riva \& Gamberini, 2000). On the contrary nowadays e-therapy has been adopted only by few enthusiasts and it is necessary a deep organizational change in Health-care providers in order to obtain a widespread use of e-therapy tools (Birch, Rigby, \& Roberts, 2000). In particular, changes in consultations and referral patterns, ways of payment, specialist support for primary healthcare, co-operation between primary and secondary healthcare, are needed. (Olsson \& Calltrop, 1999).

Although the main problem for the success of e-therapy is non-technical (Cardno, 2000), but is related to organizational changes, there are technology limitations that should be taken into account (Lou, Lin, Lin, \& Hoogstrate, 2000): the main limits are represented by insufficient image quality, low framing rate, flickering and delays that make working in front of a video terminal unattractive and tiring. However, the quality of technology in this area is increasing while costs are falling down.
To enhance the diffusion of e-therapy further research is needed. More evaluation is required of clinical outcomes, organizational effects, benefits to health-care providers and users, and quality assurance. To date the empirical research is not so strong and spread to objectively evaluate all the benefits and limits of e-therapy (Maheu \& Gordon, 2000). As clearly underlined by Maheu and Gordon, to ensure a high standard of quality "it is crucial for professional psychologists to give proper attention to empirical research and current standards of practice before attempting to deliver counseling or psychotherapy via the Internet" (p. 489). Each Internet technology requires theoretic and empirical research and, on the other side, legal and ethical guidelines to make it safe and effective as a "gold standard" clinical tool.

\section{ACKNOWLEDGMENTS}

The research was supported by the TECNOB Project (Technology for Obesity Project) funded by the "Compagnia di San Paolo", an Italian private foundation.

Many thanks to "Trafilerie di San Giovanni" and "Elettrica Brivio", Italian private enterprises, to "Manufacturas MAPREM", a Spanish private enterprise, and to $\mathrm{Mr}$. Giuseppe for their continuous and fruitful logistical support in carrying on our investigations.

\section{REFERENCES}

Alleman, J. R. (2002). Online Counseling: the Internet and the Mental Health Treatment. Psychotherapy: Theory/Research/Practice/ Training, 39(2), 199-209.

Bang, M., Timpka, T., Eriksson, H., Holm, E., \& Nordin, C. (2007). Mobile phone computing for in-situ cognitive behavioral therapy. Medinfo, 12(Pt 2), 1078-1082.

Bara-Carril, N., Williams, C. J., Pombo-Carril, M. G., Reid, Y., Murray, K., Aubin, S., et al. (2004). A preliminary investigation into the feasibility and efficacy of a CD-ROM based cognitive-behavioural selfhelp intervention for bulimia nervosa. Int J Eat Disord, 35, 538-48.

Barak, A. (1999). Psychological applications on the Internet: A discipline on the threshold of a new millennium. Appl Prev Psychol, 8(231-246).

Bauer, S., Percevic, R., Okon, E., Meermann, R., \& Kordy, H. (2003). Use of Text Messaging in the Aftercare of Patients with Bulimia Nervosa. Eur Eat Disord Rev, 11, 279-290.

Birch, K., Rigby, M., \& Roberts, R. (2000). Putting the 'tele' into health-care effectively. J Telemed Telecare, 6(Suppl 1), S113-115.

Boydell, K. M., Volpe, T., Kertes, A., \& Greenberg, N. (2007). A review of the outcomes of the recommendations made during paediatric telepsychiatry consultations. J Telemed Telecare, 13(6), 277-281.

Cardno, E. J. (2000). Managing the 'fit' of information and communication technology in community health: a framework for decision making. J Telemed Telecare, 6(Suppl 2), S6-8.

Castelnuovo, G., Buselli, C., De Ferrari, R., Gaggioli, A., Mantovani, F., Molinari, E., et al. (2004). New tools in cybertherapy: the VEPSY web site. Stud Health Technol Inform, 99, 15-35.

Castelnuovo, G., Gaggioli, A., Mantovani, F., \& Riva, G. (2003). From psychotherapy to e-therapy: the integration of traditional techniques and new communication tools in clinical settings. Cyberpsychol Behav, 6(4), 375-382.

Castelnuovo, G., Gaggioli, A., \& Riva, G. (2001). CyberPsychology meets clinical psychology: The emergence of e-therapy in mental health care. In G. Riva, Galimberti, C. (Ed.), Towards Cyberpsychology: Mind, Cogn Soc Internet Age (pp. 230-251). Amsterdam: IOS Press.

Cohen, G. E., \& Kerr, B. A. (1998). Computer mediated counseling: An empirical study of a new mental health treatment. Compute Human Serv, 15(4), 13-24.

Day, S. X., \& Schneider, P. L. (2002). Psychotherapy Using Distance Technology: A Comparison of Face-to-Face, Video and Audio Treatment. J Couns Psychol, 49(4), 499-503.

Federico, P. A. (1999). Hypermedia environments and adaptive instructions. Compute Human Behav, 15(6), 653-692. 
Frueh, B. C., Monnier, J., Grubaugh, A. L., Elhai, J. D., Yim, E., \& Knapp, R. (2007). Therapist adherence and competence with manualized cognitive-behavioral therapy for PTSD delivered via videoconferencing technology. Behav Modif, 31(6), 856-866.

Frueh, B. C., Monnier, J., Yim, E., Grubaugh, A. L., Hamner, M. B., \& Knapp, R. G. (2007). A randomized trial of telepsychiatry for posttraumatic stress disorder. J Telemed Telecare, 13(3), 142-147.

Glueckauf, R. L., Pickett, T. C., Ketterson, T. U., Loomis, J. S., \& Rozensky, R. H. (2003). Preparation for the Delivery of Telehealth Services: a Self-Study Framework for Expansion of Practice. Prof Psychol: $\operatorname{Res} \operatorname{Pr}, 34(2), 159-163$.

Glueckauf, R. L., Whitton, J. D., \& Nickelson, D. W. (2002). Telehealth: The new frontier in rehabilitation and health care. In M. J. Scherer (Ed.), Assist Technol Rehabil Psychol: Shaping an alliance (pp. 197-213). Washington, DC: American Psychological Association.

Grohol, J. M. (1999). Best Practices in e-therapy. Psychcentral.

Heinicke, B. E., Paxton, S. J., McLean, S. A., \& Wertheim, E. H. (2007). Internet-delivered targeted group intervention for body dissatisfaction and disordered eating in adolescent girls: a randomized controlled trial. J Abnorm Child Psychol, 35(3), 379-391.

Jerome, L. W., \& Zaylor, C. (2000). Cyberspace: Creating a therapeutic environment for telehealth applications. Prof Psychol: Res Pr, 31(5), 478-483.

Lester, D. (2006). E-therapy: caveats from experiences with telephone therapy. Psychol Rep, 99(3), 894-896.

Liss, H. J., Glueckauf, R. L., \& Ecklund-Johnson, E. P. (2002). Research on telehealth and chronic medical conditions: critical review, key issues and future directions. Rehabil Psychol, 47, 8-30.

Ljotsson, B., Lundin, C., Mitsell, K., Carlbring, P., Ramklint, M., \& Ghaderi, A. (2007). Remote treatment of bulimia nervosa and binge eating disorder: a randomized trial of Internet-assisted cognitive behavioural therapy. Behav Res Ther, 45(4), 649-661.

Looi, J., \& Raphael, B. (2007). Reflections on therapy in the era of the internet. Australas Psychiatry, 15(4), 334-337.

Lou, S. L., Lin, H. D., Lin, K. P., \& Hoogstrate, D. (2000). Automatic breast region extraction from digital mammograms for PACS and telemammography applications. Comput Med Imaging Graph, 24(4), 205-220.

Maheu, M. M., \& Gordon, B. L. (2000). Counseling and therapy on the Internet. Prof Psychol: Res Pr, 31(5), 484-489.

Manhal-Baugus, M. (2001). E-therapy: Practical, Ethical and Legal Issues. CyberPsychol Behav, 4(5), 551-563.

Metanoia. (2001a). ABC's of "Internet Therapy". Retrieved June 1, 2001, from www.metanoia.org/imhs/index.html

Mohr, D. C., Hart, S., \& Vella, L. (2007). Reduction in disability in a randomized controlled trial of telephone-administered cognitivebehavioral therapy. Health Psychol, 26(5), 554-563.

Molinari, E., \& Riva, G. (2004). Psicologia clinica dell'obesità (Clinical psychology of obesity). Bollati Boringhieri, Torino.

Myers, T. C., Swan-Kremeier, L., Wonderlich, S., Lancaster, K., \& Mitchell, J. E. (2004). The use of alternative delivery systems and new technologies in the treatment of patients with eating disorders. Int J Eat Disord, 36(2), 123-143.

Naylor, M. R., Keefe, F. J., Brigidi, B., Naud, S., \& Helzer, J. E. (2008). Therapeutic Interactive Voice Response for chronic pain reduction and relapse prevention. Pain, 134(3), 335-345.

Nickelson, D. (1998). Telehealth and the evolving health care system: strategic opportunities for professional psychology. Prof Psychol: Res $\operatorname{Pr}, 29,527-535$.

Norcross, J. C., Hedges, M., \& Prochaska, J. O. (2002). The Face of 2010: A Delphi Poll on the Future of Psychotherapy. Prof Psychol: Res $\operatorname{Pr}, 33,316-322$.

O'Reilly, R., Bishop, J., Maddox, K., Hutchinson, L., Fisman, M., \& Takhar, J. (2007). Is telepsychiatry equivalent to face-to-face psychiatry? Results from a randomized controlled equivalence trial. Psychiatr Serv, 58(6), 836-843.

Oakes, J., Battersby, M. W., Pols, R. G., \& Cromarty, P. (2008). Exposure therapy for problem gambling via Videoconferencing: a case report. J Gambl Stud, 24(1), 107-118.

Olsson, S., \& Calltrop, J. (1999). Telemedicine: a tool for organisational and structural change in healthcare. In R. Wootton (Ed.), Eur Telemed 1998/99 (pp. 26). London: Kensington: Publications Ltd.
Park, E. J. (2006). Telehealth technology in case/disease management. Lippincotts Case Manag, 11(3), 175-182.

Postel, M. G., de Jong, C. A., \& de Haan, H. A. (2005). Does e-therapy for problem drinking reach hidden populations? Am J Psychiat, 162(12), 2393.

Rawabdeh, A. A. (2007). An e-health trend plan for the Jordanian health care system: a review. Int J Health Care Qual Assur, 20(6), 516531.

Recupero, P., \& Rainey, S. E. (2005). Forensic aspects of e-therapy. J Psychiatr Pract, 11(6), 405-410.

Recupero, P. R., \& Rainey, S. E. (2006). Characteristics of e-therapy web sites. J Clin Psychiatry, 67(9), 1435-1440.

Reese, J. R., Conoley, C. W., \& Brossart, D. F. (2002). Effectiveness of Telephone Counseling: A Field-Based Investigation. J Couns Psychol, 49(2), 233-242.

Riva, G. (2000). Design of clinically oriented virtual environments: a communicational approach. Cyberpsychol Behav, 3(3), 351-357.

Riva, G. (2001). Shared Hypermedia: Communication and interaction in Web-based learning environments. J Educ Comput Res, 25(3), 205226.

Riva, G. (2005). Virtual reality in psychotherapy: review. Cyberpsychol Behav, 8(3), 220-230; discussion 231-240.

Riva, G., \& Gamberini, L. (2000). Virtual reality in telemedicine. Telemed $J$ E Health, 6(3), 327-340.

Riva, G., Preziosa, A., Grassi, A., \& Villani, D. (2006). Stress management using UMTS cellular phones: a controlled trial. Stud Health Technol Inform, 119, 461-463.

Robinson, P., \& Serfaty, M. (2003). Computers, E-mail and therapy in eating disorders. Eur Eat Disord Rev, 11, 210-221.

Robinson, P., \& Serfaty, M. (2008). Getting better byte by byte: a pilot randomised controlled trial of email therapy for bulimia nervosa and binge eating disorder. Eur Eat Disord Rev, 16(2), 84-93.

Robson, D., \& Robson, M. (1998). Intimacy and computer communication. Br J Guid Couns, 26, 33-41.

Shepherd, L., Goldstein, D., Whitford, H., Thewes, B., Brummell, V., \& Hicks, M. (2006). The utility of videoconferencing to provide innovative delivery of psychological treatment for rural cancer patients: results of a pilot study. J Pain Symptom Manage, 32(5), 453461.

Sherwood, N. E., Jeffery, R. W., Pronk, N. P., Boucher, J. L., Hanson, A., Boyle, R., et al. (2006). Mail and phone interventions for weight loss in a managed-care setting: weigh-to-be 2-year outcomes. Int $J$ Obes (Lond), 30(10), 1565-1573.

Shortliffe, E. H. (2000). Networking health: Prescriptions for the Internet. Washington, DC: National Academy Press.

Silk, K. R., \& Yager, J. (2003). Suggested guidelines for e-mail communication in psychiatric practice. J Clin Psychiatr, 64(7), 799-806.

Stamm, B. H. (1998). Clinical applications of telehealth in mental health care. Prof Psychol: Res Pr, 29(6), 536-542.

Suler, J. (2001). The online disinhibition effect. Retrieved Retrieved July 18, 2003, from http://www.rider.edu/ suler/psycyber/disinhibit. html

Sulzbacher, S., Vallin, T., \& Waetzig, E. Z. (2006). Telepsychiatry improves paediatric behavioural health care in rural communities. $J$ Telemed Telecare, 12(6), 285-288.

Taylor, H., \& Leitman, R. (2001). eHealth traffic critically dependent on search engines and portal. Health Care News, 1(13), 1-3.

Todder, D., \& Kaplan, Z. (2007). Rapid eye movements for acute stress disorder using video conference communication. Telemed $J E$ Health, 13(4), 461-463.

Ugarriza, D. N., \& Schmidt, L. (2006). Telecare for women with postpartum depression. J Psychosoc Nurs Ment Health Serv, 44(1), 37-45.

Urness, D., Wass, M., Gordon, A., Tian, E., \& Bulger, T. (2006). Client acceptability and quality of life--telepsychiatry compared to inperson consultation. J Telemed Telecare, 12(5), 251-254.

VandenBos, G. R., \& Williams, S. (2000). The Internet Versus the Telephone: What Is Telehealth, Anyway? Prof Psychol: Res Pr, 31(5), 490-492.

Wangberg, S. C., Gammon, D., \& Spitznogle, K. (2007). In the eyes of the beholder: exploring psychologists' attitudes towards and use of etherapy in Norway. Cyberpsychol Behav, 10(3), 418-423. 
Wiederhold, B. K., \& Wiederhold, M. D. (2003). Three-year follow-up for virtual reality exposure for fear of flying. Cyberpsychol Behav, 6(4), 441-445.

Williams, C. J. (2003). New Technologies in Self-help: Another Effective Way to Get Better? Eur Eat Disord Rev, 11(3), 170-182.

Yager, J. (2003). E-mail Therapy for Anorexia Nervosa: Prospects and Limitations Eur Eat Disord Rev, 11(3), 198-209.
Yellowlees, P. M., Hilty, D. M., Marks, S. L., Neufeld, J., \& Bourgeois, J. A. (2008). A retrospective analysis of a child and adolescent eMental Health program. J Am Acad Child Adolesc Psychiatr, 47(1), 103-107.

Zabinsky, M. F., Celio, A. A., Joy Jacobs, M., Manwaring, J., \& Wilfley, D. E. (2003). Internet-based Prevention of Eating Disorders. Eur Eat Disord Rev, 11(3), 183-197.

(C) Gianluca Castelnuovo; Licensee Bentham Open.

This is an open access article distributed under the terms of the Creative Commons Attribution License (http://creativecommons.org/licenses/by/2.5/), which permits unrestrictive use, distribution, and reproduction in any medium, provided the original work is properly cited. 